Clinical Biomechanics

1st Submission date: August 08, 2014

$1^{\text {st }}$ Revised Submission: December 23, 2014

$2^{\text {nd }}$ Revised Submission: March 7, 2015

\title{
Tensile Properties of the Transverse Carpal Ligament and Carpal Tunnel Complex
}

$1 \quad{ }^{1,2}$ Ukadike C. Ugbolue, ${ }^{2,3}$ Magnus K. Gislason, ${ }^{2}$ Mark Carter, ${ }^{4,5}$ Quentin A. Fogg, ${ }^{2}$ Philip E. Riches, and ${ }^{2}$ Philip J. Rowe.

${ }^{1}$ Biomechanics Laboratory, School of Science, Institute for Clinical Exercise \& Health Science, University of the West of Scotland, Hamilton, UK

${ }^{2}$ Department of Biomedical Engineering, University of Strathclyde, Glasgow, UK

${ }^{3}$ Biomedical Engineering, School of Science and Engineering, Reykjavik University, Iceland

${ }^{4}$ School of Life Sciences, College of Medical, Veterinary and Life Sciences, University of

9

${ }^{5}$ Department of Anatomy and Developmental Biology, Monash University, Australia

Running Title: Tensile properties of the Transverse Carpal Ligament - carpal tunnel complex

\section{Corresponding author}

Ukadike C Ugbolue

10 School of Science,

11 Biomechanics Laboratory,

12 Institute for Clinical Exercise \& Health Science,

13 University of the West of Scotland,

14 Hamilton, ML3 0JB,

15 United Kingdom

Phone: +44 (0)1698 283100 Ext 8284

Email: u.ugbolue@uws.ac.uk

17 Abstract word count: 247 words (But 269 words without Abbreviations)

18 Main text (excluding references, equations and legends): 3997 words

19

20 
1 Abstract

3 Background

4 A new sophisticated method that uses video analysis techniques together with a Maillon Rapide

5 Delta to determine the tensile properties of the transverse carpal ligament-carpal tunnel complex

6 has been developed.

7 Methods

8 Six embalmed cadaveric specimens amputated at the mid-forearm and aged (Mean (SD)): 82

9 (6.29) years were tested. The six hands were from three males (four hands) and one female (two

10 hands). Using trigonometry and geometry the elongation and strain of the Transverse Carpal

11 Ligament and carpal arch were calculated. The cross-sectional area of the Transverse Carpal

12 Ligament was determined. Tensile properties of the Transverse Carpal Ligament-Carpal Tunnel

13 Complex and Load-Displacement data were also obtained. Descriptive statistics, one-way

14 ANOVA together with a Post-hoc analysis (Tukey) and t-tests were incorporated.

15 Findings

16 A novel method to determine the tensile properties of the Transverse Carpal Ligament-Carpal

17 Tunnel Complex has been developed. There were no significant differences between the original

18 Transverse Carpal Ligament width and Transverse Carpal Ligament at peak elongation $(p=$

19 0.108). There were significant differences between the original Carpal Arch width and Carpal

20 Arch width at peak elongation $(p=0.002)$. The Transverse Carpal Ligament failed either at the

21 mid-substance or at their bony attachments. At maximum deformation the peak load and

22 maximum Transverse Carpal Ligament displacements ranged from $285.74 \mathrm{~N}$ to $1,369.66 \mathrm{~N}$ and

$237.09 \mathrm{~mm}$ to $18.55 \mathrm{~mm}$ respectively. The Transverse Carpal Ligament cross-sectional area mean

24 (SD) was $27.21(3.41) \mathrm{mm}^{2}$.

\section{Interpretation}

26 Using this method the results provide useful biomechanical information and data about the 27 tensile properties of the Transverse Carpal Ligament-Carpal Tunnel Complex.

Keywords. Transverse carpal ligament, carpal tunnel complex, tensile testing, carpal tunnel, Elongation 


\section{$1 \quad 1 \quad$ Introduction}

The clinical motivation for this study is carpal tunnel syndrome which is the most common compression and frequently diagnosed peripheral nerve disorder (Pfeffer et al., 1988;

4 Sucher and Schreiber, 2014). The carpal tunnel complex comprises bones, ligaments, muscles, tendons and nerves that are all enveloped under the skin. Within the carpal tunnel complex, the Transverse Carpal Ligament (TCL) along with the hypothenar and thenar muscles are important in the stability of the carpus and form the roof of the carpal tunnel. The volar boundary of the carpal tunnel commonly referred to as the flexor retinaculum comprises a strong band of connective tissue consisting of the proximal thin antebrachial fascia, the middle thick TCL and the most distal aponeurosis between the hypothenar and thenar musculature (Brooks et al., 2003;

11 Cobb et al., 1993; Pacek et al., 2010b). Anatomically, the TCL attaches to the carpal bones 12 distally and proximally. In the distal row, the TCL attaches to the hook of hamate on the ulnar 13 side and ridge of the trapezium on the radial side. In the proximal row, the TCL attaches to the 14 pisiform bone on the ulnar side and tubercle of the scaphoid on the radial side. In addition, the 15 tendons and median nerve together with the TCL form a pulley system (Brooks et al., 2003; Fuss 16 and Wagner, 1996; Stecco et al., 2010). During finger and hand movements the median nerve 17 and tendons move in longitudinal, transverse, and volar / dorsal directions (Lopes et al., 2011; 18 Ugbolue et al., 2005; Yoshii et al., 2008). The median nerve becomes compressed as the carpal 19 tunnel contents move within the carpal tunnel (Armstrong and Chaffin, 1979; Ugbolue et al., 20 2005).

The TCL has been investigated morphologically (Pacek et al., 2010a) and its collagen fiber orientation has been characterized (Prantil et al., 2012). So far although these studies have provided an understanding of the TCL composition and behavior, more research is still necessary 24 to fully understand the biomechanical properties of the TCL. In terms of biomechanical studies 25 associated with TCL palmar-dorsal loading, the indentation method has been used by various 26 scientific investigators (Chaise et al., 2003; Holmes et al., 2011; Ugbolue, 2012; Ugbolue et al., 27 2011). Also previous studies have investigated the biomechanical properties of the TCL 28 extracted under biaxial strain (Holmes et al., 2012) and with the TCL intact using manipulation 29 and load bearing techniques (Li et al., 2009; Sucher and Hinrichs, 1998; Xiu et al., 2010). While 30 these methods have involved either excising the TCL or determining the biomechanical 
1 properties of the carpal tunnel with the TCL intact / transected, more research designs and testing

2 procedures are necessary to evaluate the TCL and carpal tunnel complex as a whole.

Although dorsally and palmarly directed forces provide insight into the load induced

4 changes to the carpal tunnel morphology, elongating the TCL to failure would inform the 'safe'

5 range of loads that could be clinically applied during attempts to increase the carpal tunnel

6 volume, and thus reduce the pressure within the carpal tunnel. The symptoms are alleviated once

7 the pressure within the carpal tunnel subsides and / or the likely swelling of the carpal tunnel

8 contents is reduced. Currently there are various treatment methods available to help alleviate

9 Carpal Tunnel Syndrome (CTS) symptoms (Zuo et al., 2015). Percutaneous balloon carpal

10 tunnel plasty, invented by Dr J Lee Berger is a novel treatment procedure that involves stretching

11 the TCL. However, from a biomechanical research perspective more research designs and testing

12 procedures potentially could help improve current treatment procedures associated with

13 stretching the TCL and ultimately increasing the volume of the carpal tunnel. The primary

14 weakness of previous methods is not the methodologies but the fact that no testing has been done

15 in terms of elongating the ligament in situ to failure.

16 Our method provides useful information that clearly shows the range of elongation of the

17 TCL and associated changes to the carpal arch width. Also, it provides valuable data pertaining

18 to what the 'safe range' of forces applied to the TCL should be, so as to prevent the likelihood of

19 additional clinical problems such as ligament rupture. Indeed, the lack of a widely accepted

20 method does not diminish the utility of the existing data, instead a standard methodology needs

21 to be established that potentially could be referred to as an acceptable gold standard all

22 researchers can associate with. Thus, despite the valuable input provided by previous studies,

23 experimentally there is still no widely accepted testing method specifically designed to evaluate

24 the tensile properties of the carpal arch (CA) and intact TCL. That is, to date there is no known

25 method that tests the TCL to failure in situ. Hence, due to the complexity of the hand and wrist

26 the need has arisen to design and develop a sophisticated method to determine the tensile

27 properties of the carpal tunnel complex. Therefore, this study describes a novel method to

28 determine the tensile properties of the TCL in situ using two dimensional video analyses together

29 with a commercial Maillon Rapide Delta (S3i Ltd, Bawtry, England, UK). 


\section{$1 \quad 2 \quad$ Method}

The study was approved by the Department of Biomedical Engineering, University of

3 Strathclyde and the Laboratory of Human Anatomy, University of Glasgow departmental ethics

4 committees. All specimens were free from any musculoskeletal and neurological disorders.

2.1 Tensile Testing Procedure

Six embalmed cadaveric specimens amputated at the mid forearm and aged $82 \pm 6.29$ years were tested. The six hands were from four individuals (two pairs and two individual were determined using a commercial Maillon Rapide Delta (S3i Ltd, Bawtry, England, UK)

10 fastened to a steel work piece (Figure 1). The Maillon Rapide Delta is similar to a Carabiner and 11 is built to transfer forces.

Figure 1: Illustration of specimen setup on Instron E10000 (Instron, Bucks, UK) Materials 17 Testing Machine. (A) Steel work piece, (B) Rectangular aluminium bar for securing specimen, 18 (C) Maillon Rapide Delta, (D) Transverse Carpal Ligament

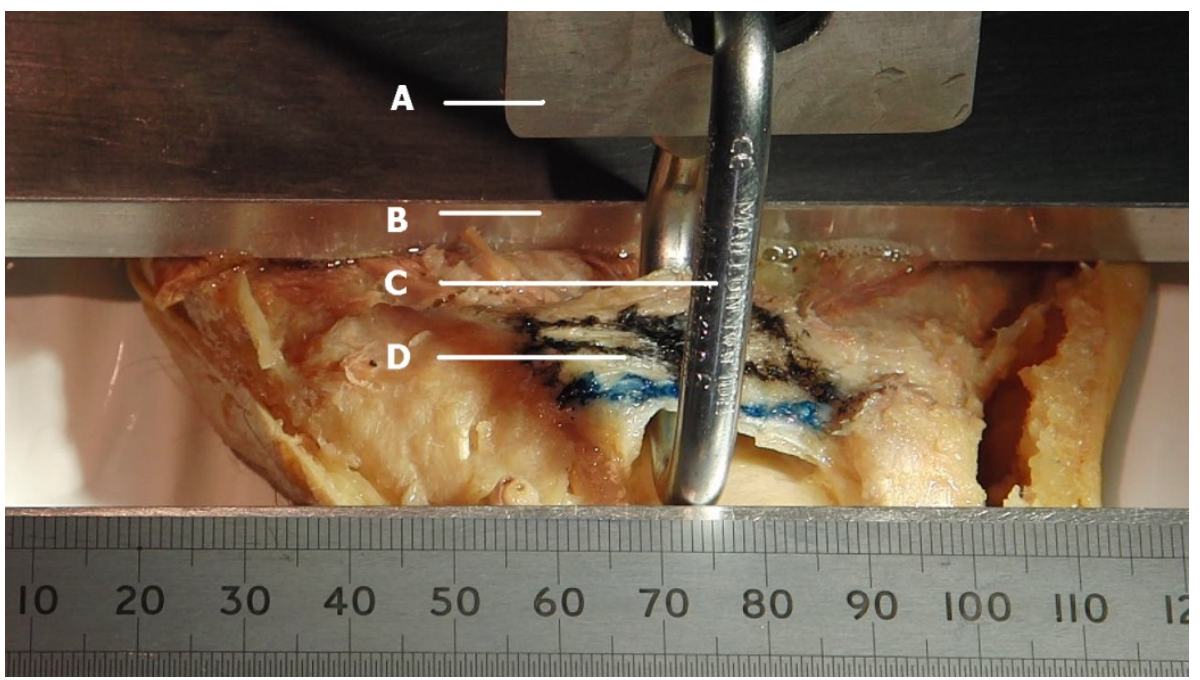

22 attached to the upper hydraulic tensile grips connected to the $1000 \mathrm{~N}$ load cell (Model: Instron

23 E10000 (Instron, Bucks, UK)), of the Instron Materials Testing Machine. The testing machine

24 was operated by a personal computer running the High Load Materials Testing Machine Instron 25 E10000 Bluehill Software package. 
Prior to testing, each specimen was prepared by exposing the TCL with carpal tunnel

2 contents removed. The thenar and hypothenar muscles together with all other surrounding soft

3 tissue such as fat and muscle fascia were removed. TCL anthropometric measurements of the

4 specimens were obtained. The lower hydraulic tensile grip was disconnected and removed to

5 provide room for the custom made aluminium specimen platform (Figure 2).

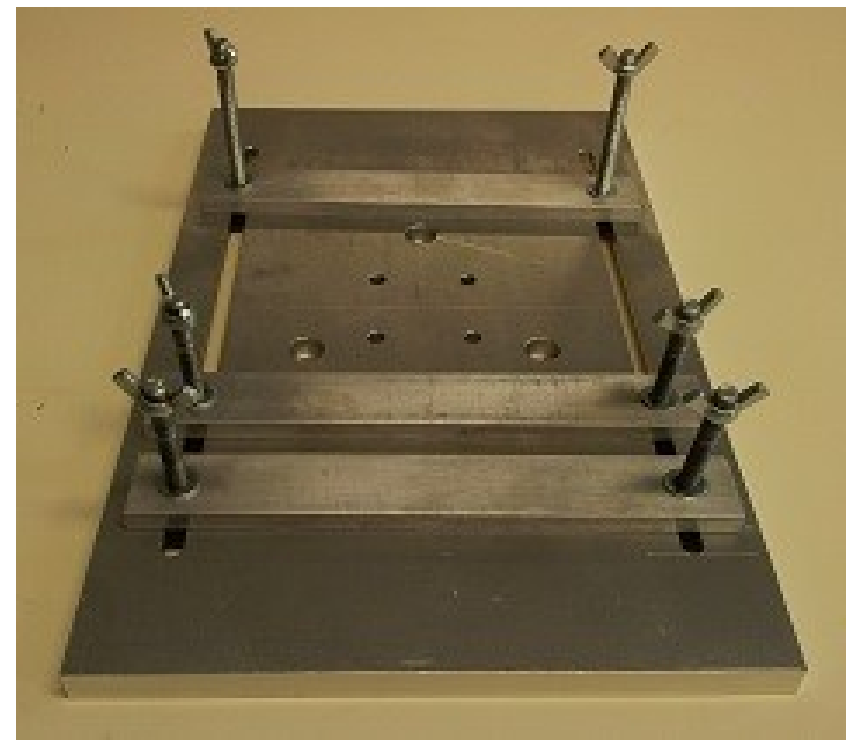

8 Figure 2: Custom made aluminium specimen platform apparatus (with three rectangular bars for 9 securing the specimen). Dimensions: overall width: $250 \mathrm{~mm}$; length: $400 \mathrm{~mm}$; depth: 15mm; bolt 10 grooves: $25 \mathrm{~mm}$ indent from side, $300 \mathrm{~mm}$ length, $8 \mathrm{~mm}$ width. Restraining bars (x3): width: $1132 \mathrm{~mm}$; length: $233 \mathrm{~mm}$; depth: $10 \mathrm{~mm}$. Bolts: $8 \mathrm{~mm}$ threaded bolts with wing nuts.

The custom made aluminium specimen platform was fastened to the base of the Instron 14 E10000 Materials Testing Machine. The specimen was placed on the aluminium platform. The 15 Maillon Rapide Delta (S3i Ltd, Bawtry, England, UK) was looped around the TCL to enable the 16 application of the palmarly directed forces without the TCL slipping free from the device. The 17 Maillon Rapide Delta (S3i Ltd, Bawtry, England, UK) was then attached to the upper hydraulic 18 tensile grips connected to the $1000 \mathrm{~N}$ load cell. The hand specimen was lying supinated with the 19 wrist in the neutral position i.e. $0^{\circ}$ flexion and $0^{\circ}$ extension. The specimen was adjusted until the 20 TCL was aligned and perpendicular to the Maillon Rapide Delta (S3i Ltd, Bawtry, England, $21 \mathrm{UK}$ ). Once the specimen was aligned, it was secured in position via three rectangular aluminium 22 bars that were tightened using two M8 threaded bolts and wing nuts per bar. The placement of 23 the three rectangular aluminium bars was standardised. The first bar was placed distal to the 
1 palmar digital crease. The second bar was placed proximal to the distal palmar crease and 2 holding down the phalanges of the thumb. The third bar was placed proximal to the wrist crease.

3 The specimens were tightly fastened and secured to the custom made aluminium specimen

4 platform apparatus. This was confirmed when no volar or side to side movements were observed 5 prior to embarking on the pre-conditioning cycle.

The test protocol started with a preconditioning cycle where the specimen underwent 10 cycles of $0.5 \mathrm{~N}$ loading at a rate of $2 \mathrm{~Hz}$. The experimental procedure entailed raising the 8 Maillon Rapide Delta (S3i Ltd, Bawtry, England, UK) and steel work piece unit until contact was made with the inside surface of the TCL and a load of $0.5 \mathrm{~N}$ was recorded. The load cell was subsequently tared and a deformation rate of $20 \mathrm{~mm} / \mathrm{s}$ was used to deform the TCL until a drop

11 off load was detected which was categorized as failure of the ligament. Indeed, this testing 12 procedure being unique had no reference point with regards to a choice of suggested deformation 13 rates. A series of tests were performed in the laboratory prior to arriving at the $20 \mathrm{~mm} / \mathrm{s}$ 14 deformation rate. A selection of deformation rates were chosen for the pilot test. Upon 15 completion of the testing, a deformation rate of $5 \mathrm{~mm} / \mathrm{s}$ was too slow. The deformation rate was 16 then doubled to $10 \mathrm{~mm} / \mathrm{s}$ which again was too slow. For both deformation rates (at $5 \mathrm{~mm} / \mathrm{s}$ and $1710 \mathrm{~mm} / \mathrm{s}$ ) the Maillon Rapide Delta kept on slipping away from the centre of the ligament which 18 was set to be in line with the steel work piece unit attached to the upper hydraulic tensile grips 19 connected to the $1000 \mathrm{~N}$ load cell (Model: Instron E10000 (Instron, Bucks, UK)). Once the 20 deformation rate was increased to $20 \mathrm{~mm} / \mathrm{s}$ the slipping motion of the Maillon Rapide Delta 21 reduced considerably as the soft tissue underwent deformation. Initially, a customised D'Shackle 22 was used to determine the tensile properties of the intact TCL. After a few trials the authors came 23 to realise it was not a good fit for specimens with smaller sized carpal tunnels and TCL lengths 24 greater than 20mm; hence the Maillon Rapide Delta (S3i Ltd, Bawtry, England, UK) was 25 preferred. This unique approach uses a Maillon Rapide Delta (S3i Ltd, Bawtry, England, UK) 26 attached to a steel work piece that is secured between jaws of the upper hydraulic tensile grips of 27 the Instron E10000 (Instron, Bucks, UK) Materials Testing Machine. The preconditioning cycle 28 loading conditions were low primarily to preserve the integrity of the TCL insertion sites prior to 29 deformation. The TCL elongation and ligament strain were calculated. Stiffness was calculated 30 as the slope of the linear portion of the Load - Displacement graph. Using the Excel software 
1 tools (Microsoft Excel 2010) $\mathrm{R}^{2}$ was $\geq 0.92$ for all trials. The cross-sectional area (A) was

2 estimated as

$$
\mathrm{A}=M L \times\left(\frac{D T+P T}{2}\right)
$$

4 Where ML $=$ TCL Mid Length, DT $=$ TCL Distal Thickness, PT $=$ TCL Proximal Thickness

\subsection{Calculation of ligament elongation and strain}

The ligament elongation and strain was calculated for the tensile test. A two dimensional model has been developed that incorporates prior knowledge from a simplified two dimensional

8 model that assumes linear elastic TCL stretch. This simplified model uses calculations applicable

9 to a flat head indenter (Holmes et al., 2011). Diagrammatically the TCL is shown in Figure 3 10 prior to undergoing any deformation.

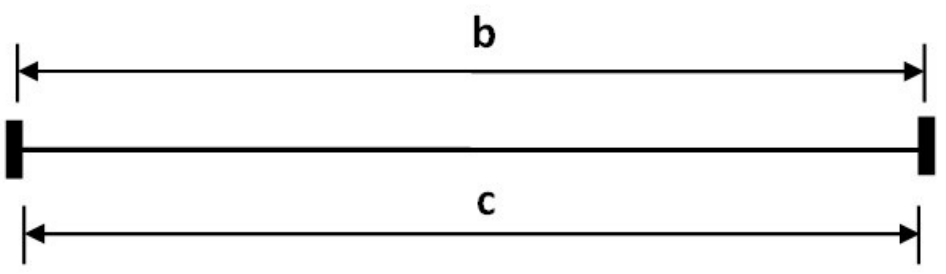

12
Figure 3: Schematic of the intact TCL showing (a) Direction of stretch / elongation (tensile deformation), (b) Original width of the TCL $\left(\boldsymbol{b}_{T C L}\right)$ and (c) Original width of the CA $\left(\boldsymbol{b}_{C A}\right)$

Three TCL anthropometric measurements of the specimens were obtained using an electronic 150mm LCD digital Vernier Caliper (Gizmo Deals Ltd, Northamptonshire, UK). The width of the TCL was calculated as the average of the dimensions of the TCL distal width, TCL proximal width and TCL mid width. Ligament strain was calculated as the increase in TCL width divided by the original width of the TCL. The term 'Original' refers to the relaxed or pre-loaded condition of the ligament. Both the TCL width and CA width are terms used to define the carpal tunnel complex. The original TCL width $\left(\boldsymbol{b}_{T C L}\right)$ represents the width of the external surface 
1 dimension of the TCL (volar side) at rest while the original CA width $\left(\boldsymbol{b}_{\boldsymbol{C A}}\right)$ represents the

2 internal dimension of the TCL (dorsal side) at rest parallel to its surface.

\section{$4 \quad 2.3 \quad$ Tensile test using a commercial Maillon Rapide Delta}

5 Video recordings of the tensile test were captured using a high speed video camera (EX-FH20

6 EXILIM, Casio, USA) mounted on a tripod. From the captured experiment video recordings the

7 percentage of the Maillon Rapide Delta (S3i Ltd, Bawtry, England, UK) in contact with the TCL

8 was consistent for all specimens. The Maillon Rapide Delta (S3i Ltd, Bawtry, England, UK) was

9 approximately $33 \%$ in contact with the TCL upon TCL elongation and tensile deformation. The

10 term TCL elongation and tensile deformation refer to the elasto-plastic deformation the ligament

11 undergoes during the tensile test. Using the commercially available ProTrainer system (Sports

12 Motion Inc $\AA$, Cardiff, CA) software tools, a clock face the size of the circumference of the

13 Maillon Rapide Delta located under the TCL was generated. Using the angle tool three points

14 were marked on the generated circle (Figure 4). The marginal contact points occurring at the

15 proximal end of the TCL were then manually digitized. Based on unpublished reliability studies

16 performed within our Biomechanics Laboratory, the accuracy of the marginal contacts between

17 the TCL and Maillon Rapide Delta were determined. The video camera was placed perpendicular

18 to the TCL such that the vantage point focused on the sagittal place of the TCL. This camera set

19 up procedure was standardised for all specimens to ensure the off-axis angle of the video in

20 relation to the horizontal portion of the MRD was reduced. 


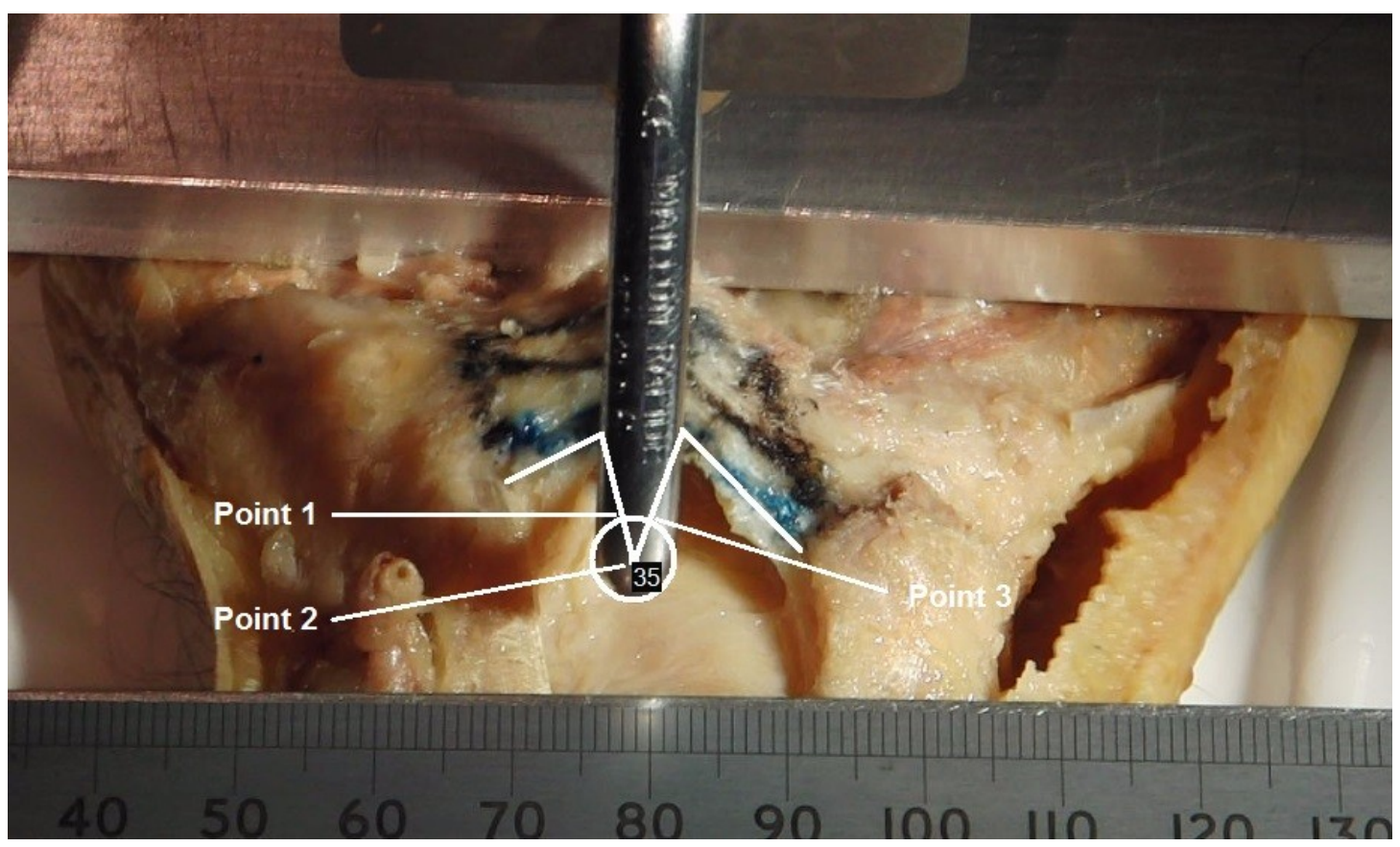

3 Figure 4: Picture showing angle formed after digitisation. Point 1: Marginal TCL contact with 4 Maillon Rapide Delta (Left Side), Point 2: Centre of the clock face, Point 3: Marginal TCL 5 contact with Maillon Rapide Delta (Right Side). $\theta$ was defined as the angle formed with respect 6 to the three points. $\theta$ in this case is $35^{\circ}$.

7 Based on a simplified model by Holmes et al, 2011 a novel method that applies

8 mathematical principles has recently been developed to calculate the tensile ligament elongation

9 and strain. From Figure 5, the following calculations were applied to determine the tensile 10 ligament elongation and strain. 


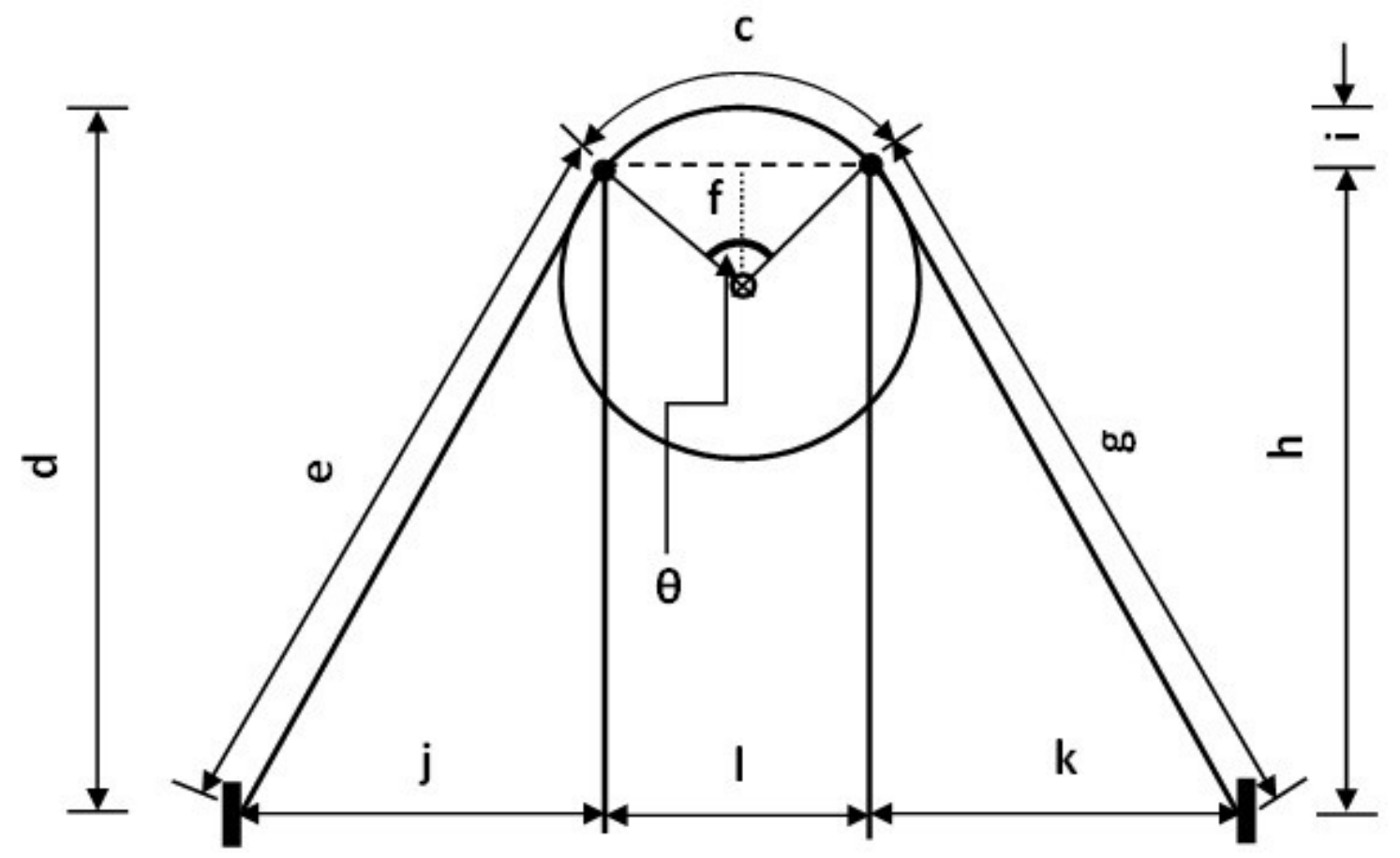

2 Figure 5: Illustration of the TCL undergoing elongation and tensile deformation. (c) Arc length, (d) Maillon Rapide Delta displacement, (e) TCL width on either side of Maillon Rapide Delta at peak ligament elongation, (f) Apothem, (g) Same as (e), (h) Maillon Rapide Delta displacement with respect to chord length, (i) Difference between the radius and apothem, (j) Horizontal ligament width with respect to TCL width (e), (k) Horizontal ligament width with respect to TCL width (g), and (1) Chord length.

9 The Maillon Rapide Delta used had a radius $\mathbf{r}$ of $5 \mathrm{~mm}$. The angle $\boldsymbol{\theta}$ was determined using the 10 angle tool in the ProTrainer system (Sports Motion Inc ${ }^{\circledR}$, Cardiff, CA) software. The arc length c 11 (from Figure 5) was calculated using the formula

$$
\mathrm{c}=r \theta
$$

14 The apothem $\mathbf{f}$ was determined by applying trigonometry, where

$$
f=r \cos \left(\frac{\theta}{2}\right)
$$

17 and 


$$
i=r-f
$$

2

3

8 From the experiments at peak ligament elongation 13 Thus,

23 So that,

At peak ligament elongation $\mathrm{d}$,

$$
h=d-i
$$

$$
b \neq(j+l+k)
$$

$$
n=j+l+k
$$

$$
n-l=j+k
$$

The chord length $\boldsymbol{l}$ can be calculated as

$$
l=2 r \sin \left(\frac{\theta}{2}\right)
$$

Assuming $\boldsymbol{j}$ and $\boldsymbol{k}$ are symmetrical, then

$$
j=k
$$

$$
n-l=2 j
$$

$$
j=k=\frac{n-l}{2}
$$

But

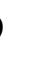

$$
e=g=\sqrt{h^{2}+j^{2}}
$$

From the video recordings the CA width at peak ligament elongation $\boldsymbol{n}$ can be extracted.

If $\mathbf{e}$ and $\mathbf{g}$ were asymmetrical then both lengths will have to be solved for independently. 
1 Therefore, total ligament width due to elongation $\boldsymbol{P}_{T C L-e}$ is given as:

$$
P_{T C L-e}=e+c+g
$$

Hence ligament strain due to elongation $\boldsymbol{e}_{\boldsymbol{T C L}-\boldsymbol{e}}$ can be calculated as

$$
e_{T C L-e}=\frac{P_{T C L-e}-b_{T C L}}{b_{T C L}}
$$

And carpal arch strain due to elongation $\boldsymbol{e}_{\boldsymbol{C A}-\boldsymbol{e}}$ can be calculated as

$$
e_{C A-e}=\frac{n-b_{C A}}{b_{C A}}
$$

Descriptive statistics were incorporated into the study. TCL tensile properties were determined. Pearson correlation was applied to ascertain whether there was a relationship between the TCL cross-sectional area and the TCL and CA elongation and strain measurements. One-way ANOVA together with a Post-hoc analysis (Tukey) was applied to distinguish between the CA width different levels of deformation. T-tests were performed to compare the differences between elongation and strain measurements of the TCL and CA. P value was set to 0.05 .

\section{Results}

A unique method that incorporates geometric and trigonometric applications has been developed to determine the elongation and strain of the CA and TCL. In terms of the level of deformation caused to the TCL, using the Maillon Rapide Delta (S3i Ltd, Bawtry, England, UK), there were no significant differences between the original TCL width and TCL at peak elongation ( $p=$ 0.108). The mean difference between the original TCL width and TCL at peak elongation was $5.09 \mathrm{~mm}$. The mean difference between the CA width at peak elongation and TCL at peak elongation was $14.18 \mathrm{~mm}$. The results can be seen in Table 1. Also, the mean difference between the CA strain and TCL peak ligament strain was 0.18 . There was a significant difference at peak elongation between the CA width and TCL width $(p=0.007)$. The CA strain was statistically significantly different from the TCL peak ligament strain $(p=0.006)$. From the one-way Anova the main effect showed significant differences between the CA width, the CA width at peak 
1 elongation and CA width after TCL break $(p<0.0001)$. The post-hoc comparisons showed 2 significant differences between the original CA width and CA width at peak elongation $(p=$ 3 0.002). Though there were significant differences between the original CA width and CA width 4 after break $(p=0.002)$, there were no significant differences between the CA width at peak 5 elongation and CA width after break $(p=0.29)$.

6

Table 1 Representation of TCL and Carpal Arch data under tension. 8

\begin{tabular}{|c|c|c|c|c|c|c|c|}
\hline \multirow[b]{2}{*}{ Specimens } & \multirow[b]{2}{*}{$\begin{array}{l}\text { Original } \\
\text { Width of } \\
\text { the TCL } \\
(\mathrm{mm})\end{array}$} & \multicolumn{2}{|c|}{ TCL Under Tension } & \multirow[b]{2}{*}{$\begin{array}{c}\text { Original } \\
\text { Carpal } \\
\text { Arch } \\
\text { Width } \\
(\mathbf{m m})\end{array}$} & \multirow[b]{2}{*}{$\begin{array}{l}\text { Carpal Arch } \\
\text { Width at } \\
\text { Peak } \\
\text { Elongation } \\
\text { (mm) }\end{array}$} & \multirow[b]{2}{*}{$\begin{array}{c}\text { Carpal } \\
\text { Arch } \\
\text { Width after } \\
\text { TCL Break } \\
\text { (mm) }\end{array}$} & \multirow{2}{*}{$\begin{array}{c}\text { Carpal } \\
\text { Arch Strain }\end{array}$} \\
\hline & & $\begin{array}{c}\text { Peak } \\
\text { Ligament } \\
\text { Elongation } \\
(\mathrm{mm})\end{array}$ & $\begin{array}{c}\text { Peak } \\
\text { Ligament } \\
\text { Strain }\end{array}$ & & & & \\
\hline $1 \mathrm{~L}_{\mathrm{m}}$ & 25.22 & 32.40 & 0.28 & 17.00 & 16.00 & 20.00 & -0.06 \\
\hline $2 \mathrm{R}_{\mathrm{m}}$ & 27.97 & 21.64 & 0.23 & 19.00 & 16.00 & 20.00 & -0.18 \\
\hline $3 \mathrm{~L}_{\mathrm{m}}$ & 22.03 & 28.16 & 0.28 & 19.00 & 17.00 & 22.00 & -0.11 \\
\hline $4 \mathrm{R}_{\mathrm{f}}$ & 22.93 & 26.56 & 0.16 & 20.00 & 19.00 & 22.00 & -0.05 \\
\hline $5 \mathrm{~L}_{\mathrm{f}}$ & 26.21 & 33.21 & 0.27 & 18.00 & 16.00 & 19.00 & -0.11 \\
\hline $6 \mathrm{~L}_{\mathrm{m}}$ & 27.20 & 40.14 & 0.48 & 15.00 & 13.00 & 16.00 & -0.13 \\
\hline Mean & 25.26 & 30.35 & 0.28 & 18.00 & 16.17 & 27.75 & -0.10 \\
\hline $\mathrm{SD}$ & 2.36 & 6.38 & 0.11 & 1.79 & 1.94 & 2.01 & 0.04 \\
\hline
\end{tabular}

L - Left Hand; R - Right Hand, m- male, f - female.

10 The TCL failed either at the mid-substance or at their bony attachments. Five specimens failed at

11 their bone attachments of which four (Specimens 1, 2, 4 and 6) failed completely on the radial

12 side of the TCL. One specimen (Specimen 5) had a combined failure that started at the hamate

13 bony attachment point and continued at the mid-substance. The results further provide useful

14 information and data about the variation of the TCL tensile properties when tested in the volar-

15 dorsal direction (Figure 6). At maximum deformation the peak load and maximum TCL

16 displacements ranged from $285.74 \mathrm{~N}$ to $1,369.66 \mathrm{~N}$ and $7.09 \mathrm{~mm}$ to $18.55 \mathrm{~mm}$ respectively. The

17 load at tensile strength ranged from $272.09 \mathrm{~N}$ to $1293.36 \mathrm{~N}$ and the ultimate tensile strength mean 18 (SD) was $23.99(10.68) \mathrm{Nmm}^{-2}$. The TCL cross-sectional area mean (SD) was $27.21(3.41) \mathrm{mm}^{2}$

19 and TCL stiffness was $71.66(17.31) \mathrm{Nmm}^{-1}$. See Table 2. There was no correlation between the

20 TCL cross-sectional area and the peak ligament elongation $\left(\mathrm{R}^{2}=0.118\right)$, peak ligament strain $\left(\mathrm{R}^{2}\right.$

$21=0.245)$, carpal arch width at peak elongation $\left(R^{2}=0.153\right)$ and carpal arch strain $\left(R^{2}=0.024\right)$. 
2 Table 2 Data Summary of TCL Tensile Properties.

\begin{tabular}{|c|c|c|c|c|c|}
\hline \multirow{3}{*}{ Specimens } & $\begin{array}{c}\text { TCL Cross- } \\
\text { Sectional } \\
\text { Area } \\
\left(\mathbf{m m}^{2}\right)\end{array}$ & $\begin{array}{c}\text { Displacement } \\
\text { at Peak Load }\end{array}$ & $\begin{array}{c}\text { Peak Load at } \\
\text { Maximum } \\
\text { Deformation }\end{array}$ & $\begin{array}{c}\text { Load at } \\
\text { Tensile } \\
\text { Load } \\
\mathbf{( N )}\end{array}$ & $\begin{array}{c}\text { Ultimate } \\
\text { Tensile } \\
\text { Strength } \\
\left(\mathbf{N m m}^{-2}\right)\end{array}$ \\
\hline $1 \mathrm{~L}_{\mathrm{m}}$ & 30.59 & 13.74 & 527.89 & 515.80 & 16.86 \\
$2 \mathrm{R}_{\mathrm{m}}$ & 26.42 & 7.09 & 684.09 & 632.37 & 23.93 \\
$3 \mathrm{~L}_{\mathrm{m}}$ & 26.79 & 10.95 & 699.82 & 631.12 & 23.56 \\
$4 \mathrm{R}_{\mathrm{f}}$ & 26.85 & 9.07 & 285.74 & 272.09 & 10.13 \\
$5 \mathrm{~L}_{\mathrm{f}}$ & 21.62 & 14.20 & 607.88 & 600.16 & 27.76 \\
$6 \mathrm{~L}_{\mathrm{m}}$ & 31.02 & 18.55 & 1369.66 & 1293.36 & 41.70 \\
\hline
\end{tabular}

4 L - Left Hand; R - Right Hand, m- male, f - female. 


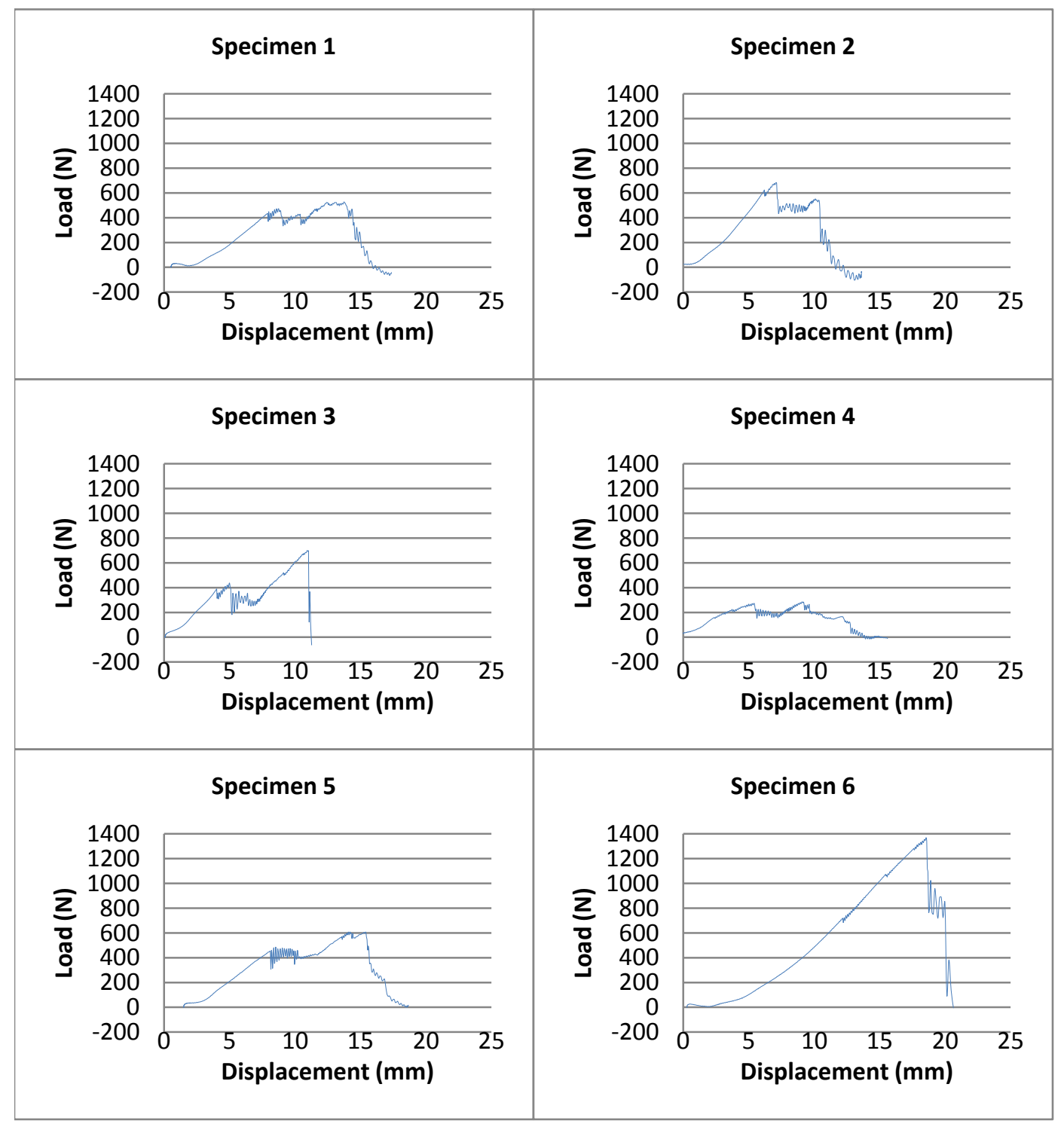

$3 \quad$ Figure 6: Load - Displacement curve for all six specimens. 


\section{Discussion}

A new sophisticated method has been developed that can be used to determine the tensile properties of the TCL in situ. This has all along been challenging to researchers investigating the biomechanical properties and role of the TCL from a computational (Guo et al., 2009; Li et al., 2009; Main et al., 2012) and experimental (Garcia-Elias et al., 1989; Tengrootenhuysen et al., 2009; Xiu et al., 2010) perspective. This paper describes the first approach taken towards determining tensile properties of the TCL using a commercial Maillon Rapide Delta. This methodology has been validated and is capable of generating highly reliable and repeatable data. (Ugbolue et al., 2013) Previous methods have investigated the expansion or lengthening of the TCL and carpal tunnel complex, however, none of these methods report the tensile properties of the TCL in situ. This method tests the TCL to failure and invariably provides data on the elongation and strain of the carpal arch and TCL. Although, standard bone-ligament-bone tests are well established methodologies, the introduction of this new method evaluates the TCL carpal tunnel complex and adds useful information to the knowledge database pertaining to the biomechanical properties of the TCL and carpal tunnel complex. With precautionary measures observed during dissection, this new method involved carefully and meticulously exposing the TCL by removing all the soft tissues (fat, muscles, muscle fascia, etc) overlying the TCL.

Further care was taken to ensure the TCL insertion sites and integrity of its bony attachments remained intact. The constituent parts of the carpal tunnel complex were not isolated. As previous studies have assessed the TCL, either excised or as a bone-ligament-bone complex, making comparisons was somewhat difficult. The specimens were not young and were aged (mean (SD)): 82 (6.29) years. At present, we are unaware of any study that has investigated the differences in biomechanical properties of the TCL between young adults and elderly human

24 subjects. Data on changes to the anterior cruciate ligament (ACL) material properties with 25 respect to age is available. Woo and colleagues (1991) showed a considerable difference in ACL 26 tensile strength with respect to age: $2160 \mathrm{~N}$ for younger specimens (22-35 years) and $658 \mathrm{~N}$ in 27 older specimens (60-97 years) (Woo et al., 1991). In terms of the categories used by Woo et al 28 (1991), all our specimens fall into the older specimen category. These findings may be applicable to the current study; however there is no direct evidence to suggest the TCL follows similar 30 trends with age. Based on the current study results and sample size used, it is not enough to 31 speculate that the onset of CTS and gender differences affect tensile strength and elongation 
measurements. Although the sites of failure for some of the specimens were comparable the 2 failure patterns produced were different. Figure 6 shows the variations in the Load 3 Displacement curves across the six specimens. These variations could be attributed to the 4 subjective judgement involved during the dissection process while identifying the precise 5 margins of the TCL tissue. Furthermore other factors such as cross-sectional area, variations in the sizes of the carpal tunnel and dimensions of the TCL and CA could also have influenced the differences in the Load - Displacement curve profile between the specimens. Also since the 8 carpal bones were neither isolated nor monitored it is possible the displacement of the carpal bones may have also contributed to the entire elongation of the TCL. Although, the anthropometric measurements and cross-sectional area may have influenced the TCL under

11 tension outcome measures i.e. Load - Displacement curve profile between the specimens, there 12 was no correlation between the TCL cross-sectional area and TCL and CA elongation and strain 13 measurements $\left(R^{2} \leq 0.245\right)$. Also there was no trend in the data to suggest that specimens with 14 smaller cross-sectional areas produced smaller maximum displacements (at peak loads), smaller 15 peak loads at maximum deformation, smaller loads at tensile strength and smaller ultimate 16 tensile strength values. However, specimen 6 possessed the highest cross-sectional area with 17 corresponding high values for the maximum displacement (at peak load), peak load at maximum 18 deformation, load at tensile strength and ultimate tensile strength. Based on our results the peak 19 load at maximum deformation lies between $285.74 \mathrm{~N}$ and $1369.66 \mathrm{~N}$. This load deformation range 20 suggests that forces applied outside of this representative 'safe range' may cause additional 21 problems or complications to the TCL and soft tissues of the hand. Also since the other 22 parameters do not have any outliers the mean could be considered as a 'safe measurement' 23 representative of the tensile properties reported in Table 2.

24 Due to the novelty of the methodology few comparisons are made with other studies 25 regarding the results. Comparing the anthropometric measurement average values to that 26 reported by Holmes et al, 2011, the TCL width at rest and peak loading had a mean difference of $274.04 \mathrm{~mm}$ and $0.95 \mathrm{~mm}$ respectively. These variations were small considering specimen variability 28 is an inherent factor considered and incorporated into cadaver related research designs. TCL 29 tensile testing has predominantly been performed in the radial / ulnar direction with very little 30 testing done until recently in the volar / dorsal direction (Main et al., 2012). Previous studies 31 have investigated TCL tensile testing using manipulation and load bearing techniques (Sucher 
1 and Hinrichs, 1998; Xiu et al., 2010). Sucher and colleagues demonstrated a maximal TCL 2 elongation of $3.7 \mathrm{~mm}$ of the carpal arch in female cadavers using a $10 \mathrm{~N}$ static load compared to a 3 carpal arch width of $1.83 \mathrm{~mm}$ in our study which used a combination of both genders. Xiu et al 4 (2010) showed that an intact carpal arch width increased by about $1 \mathrm{~mm}$ when the TCL was 5 subjected to an applied force of $10 \mathrm{~N}$ in the outward direction. Aside from load bearing and manipulation techniques, bone-ligament-bone tensile testing methods have also been adopted (Garcia-Elias et al., 1989). Lin and associates showed no significant differences in TCL tensile 8 properties between TCL samples excised from CTS patients undergoing carpal tunnel release and those samples obtained from fresh control cadaveric specimens. (Lin et al., 1983) While

10 these methods have been a valuable contribution to the tensile biomechanical assessment of the

11 TCL and CA, our method provides an efficient method to specifically evaluate the tensile properties of the intact TCL in situ.

An earlier study by Li and associates investigated the expansion of the carpal tunnel by 14 applying palmarly directed forces to the TCL from inside the carpal tunnel (Li et al., 2009). This 15 model used a custom lever device to apply known forces that generated TCL arches. In the study 16 by $\mathrm{Li}$ et al, 2009, a geometric model was also developed to clarify the relationships between the 17 TCL width, CA width, CA height and CA area. The author's model is different but provides a 18 useful approach designed to determine the tensile properties of the intact TCL. Though their methodology is different, their study reported the TCL arch length (mean (SD)) as $2.8(0.30) \mathrm{mm}$ at $10 \mathrm{~N}$ and $5.4(0.40) \mathrm{mm}$ at $200 \mathrm{~N}$ compared to $0.54(0.52) \mathrm{mm}$ at $10 \mathrm{~N}$ and $4.25(1.54) \mathrm{mm}$ at $200 \mathrm{~N}$. The differences in the results may be attributed to (a) the methodologies adopted or (b) the

22 choice of specimens i.e. fresh-frozen versus embalmed specimens. Our study used embalmed 23 specimens for the experimental tests. To date there are no ligament studies to suggest they are 24 differences in the mechanical properties between fresh-frozen versus embalmed specimens. 25 Studies on bone mechanics to determine differences between fresh-frozen and embalmed 26 specimens have been done. These studies have shown that fresh-frozen and embalmed bones 27 have similar characteristics with very low variation in mechanical strength between unpaired 28 cadaveric specimens which suggests the use of paired specimens may be unnecessary. (Topp et 29 al., 2012; Zech et al., 2006)

30 Prior to testing, the Maillon Rapide Delta was centrally aligned which left little or no 31 room for $\mathbf{e}$ and $\mathbf{g}$ to be asymmetrical. During the test care was taken to ensure the Maillon Rapide 
1 Delta was not misaligned. Although four specimens failed completely on the radial side of the

2 TCL, looking back at the videos there was no sudden shift of the Maillon Rapide Delta to the

3 radial side to suggest why the failure occurred on the radial side. Since the TCL has a role to play

4 biomechanically, the approach taken to determine the tensile properties of the TCL has clinical

5 implications that may influence treatment methods that do not involve dissecting the TCL. The

6 treatment method percutaneous balloon carpal tunnel plasty is unique and is gradually becoming

7 an acceptable treatment option available to carpal tunnel syndrome patients where the integrity

8 of the TCL is retained and the symptoms are alleviated through the stretching of the TCL. It is

9 anticipated, clinicians can leverage the valuable contents presented in this study to make

10 informed decisions regarding the treatment protocols designed to treat symptoms or help patients

11 cope with symptoms associated with carpal tunnel syndrome.

\section{Conclusion}

13 Due to the complexity of the hand anatomy, a novel method and unique testing procedure

14 that is uncomplicated and practical has been developed to determine the tensile properties of the

15 TCL and carpal tunnel complex. We anticipate this unique testing approach could be adopted by

16 researchers interested in determining the tensile properties of ligaments. Furthermore, using this

17 method of determining the tensile properties of the TCL and carpal tunnel complex would 18 provide biomechanical information computational modellers, biomechanists and clinical 19 researchers can benefit from.

\section{Acknowledgment}

22 The author will like to thank the Department of Biomedical Engineering, University of 23 Strathclyde for access to the material testing equipment.

\section{Conflict of Interest Statement}

26 The authors do not have any conflict of interest that could inappropriately influence this work. 


\section{References}

Armstrong, T.J., Chaffin, D.B., 1979. Some biomechanical aspects of the carpal tunnel. Journal of biomechanics 12, 567-570.

Brooks, J.J., Schiller, J.R., Allen, S.D., Akelman, E., 2003. Biomechanical and anatomical consequences of carpal tunnel release. Clin Biomech (Bristol, Avon) 18, 685-693.

Chaise, J., Ugbolue, U.C., Li, Z.M., 2003. Viscoelastic Properties of the Transverse Carpal Ligamant. , , Musculoskeletal Research Center Summer Research Program Annual Symposium, Pittsburgh, USA. Published in Musculoskeletal Research Center Summer Research Program Booklet, 2003. Cobb, T.K., Dalley, B.K., Posteraro, R.H., Lewis, R.C., 1993. Anatomy of the flexor retinaculum. The Journal of hand surgery 18, 91-99.

Fuss, F.K., Wagner, T.F., 1996. Biomechanical alterations in the carpal arch and hand muscles after carpal tunnel release: a further approach toward understanding the function of the flexor retinaculum and the cause of postoperative grip weakness. Clin Anat 9, 100-108.

Garcia-Elias, M., An, K.N., Cooney, W.P., 3rd, Linscheid, R.L., Chao, E.Y., 1989. Stability of the transverse carpal arch: an experimental study. The Journal of hand surgery 14, 277-282. Guo, X., Fan, Y., Li, Z.M., 2009. Effects of dividing the transverse carpal ligament on the mechanical behavior of the carpal bones under axial compressive load: a finite element study. Medical engineering \& physics 31, 188-194.

Holmes, M.W., Howarth, S.J., Callaghan, J.P., Keir, P.J., 2011. Carpal tunnel and transverse carpal ligament stiffness with changes in wrist posture and indenter size. Journal of orthopaedic research : official publication of the Orthopaedic Research Society 29, 1682-1687.

Holmes, M.W., Howarth, S.J., Callaghan, J.P., Keir, P.J., 2012. Biomechanical properties of the transverse carpal ligament under biaxial strain. Journal of orthopaedic research : official publication of the Orthopaedic Research Society 30, 757-763.

Li, Z.M., Tang, J., Chakan, M., Kaz, R., 2009. Carpal tunnel expansion by palmarly directed forces to the transverse carpal ligament. Journal of biomechanical engineering 131, 081011. Lin, R., Lin, E., Engel, J., Bubis, J.J., 1983. Histo-mechanical aspects of carpal tunnel syndrome. The Hand 15, 305-309. Lopes, M.M., Lawson, W., Scott, T., Keir, P.J., 2011. Tendon and nerve excursion in the carpal tunnel in healthy and CTD wrists. Clin Biomech (Bristol, Avon) 26, 930-936.

Main, E.K., Goetz, J.E., Baer, T.E., Klocke, N.F., Brown, T.D., 2012. Volar/dorsal compressive mechanical behavior of the transverse carpal ligament. Journal of biomechanics 45, 1180-1185. Pacek, C.A., Chakan, M., Goitz, R.J., Kaufmann, R.A., Li, Z.M., 2010a. Morphological analysis of the transverse carpal ligament. Hand (N Y) 5, 135-140.

Pacek, C.A., Tang, J., Goitz, R.J., Kaufmann, R.A., Li, Z.M., 2010b. Morphological analysis of the carpal tunnel. Hand (N Y) 5, 77-81.

Pfeffer, G.B., Gelberman, R.H., Boyes, J.H., Rydevik, B., 1988. The history of carpal tunnel syndrome. Journal of hand surgery 13, 28-34.

Prantil, R.K., Xiu, K., Kim, K.E., Gaitan, D.M., Sacks, M.S., Woo, S.L., Li, Z.M., 2012. Fiber orientation of the transverse carpal ligament. Clin Anat 25, 478-482.

Stecco, C., Macchi, V., Lancerotto, L., Tiengo, C., Porzionato, A., De Caro, R., 2010. Comparison of transverse carpal ligament and flexor retinaculum terminology for the wrist. The Journal of hand surgery 35, 746-753. 
Sucher, B.M., Hinrichs, R.N., 1998. Manipulative treatment of carpal tunnel syndrome: biomechanical and osteopathic intervention to increase the length of the transverse carpal ligament. The Journal of the American Osteopathic Association 98, 679-686.

Sucher, B.M., Schreiber, A.L., 2014. Carpal tunnel syndrome diagnosis. Physical medicine and rehabilitation clinics of North America 25, 229-247.

Tengrootenhuysen, M., van Riet, R., Pimontel, P., Bortier, H., Van Glabbeek, F., 2009. The role of the transverse carpal ligament in carpal stability: an in vitro study. Acta orthopaedica Belgica $75,467-471$.

Topp, T., Muller, T., Huss, S., Kann, P.H., Weihe, E., Ruchholtz, S., Zettl, R.P., 2012. Embalmed and fresh frozen human bones in orthopedic cadaveric studies: which bone is authentic and feasible? Acta orthopaedica 83, 543-547.

Ugbolue, U.C., 2012. Biomechanics and morphology of the carpal tunnel complex. In: 2012 Research Day at the University of Strathclyde, Glasgow, UK: 7 June 2012.

Ugbolue, U.C., Carter, M., Fogg, Q., Riches, P.E., Rowe, P.J., 2011. Biomechanical properties of the carpal tunnel complex. In: 2011 UK Hand / Wrist / Shoulder Meeting, 1-2 September 2011, Glasgow, U.K.

Ugbolue, U.C., Gislason, M.K., Fogg, Q.A., Carter, M., Riches, P.E., Rowe, P.J., 2013. A pilot study to determine the tensile properties of the transverse carpal ligament, XXIV Congress of the International Society of Biomechanics (XV Brazilian Congree of Biomechanics). Ugbolue, U.C., Hsu, W.H., Goitz, R.J., Li, Z.M., 2005. Tendon and nerve displacement at the wrist during finger movements. Clin Biomech (Bristol, Avon) 20, 50-56.

Woo, S.L., Hollis, J.M., Adams, D.J., Lyon, R.M., Takai, S., 1991. Tensile properties of the human femur-anterior cruciate ligament-tibia complex. The effects of specimen age and orientation. The American journal of sports medicine 19, 217-225.

Xiu, K.H., Kim, J.H., Li, Z.M., 2010. Biomechanics of the transverse carpal arch under carpal bone loading. Clin Biomech (Bristol, Avon) 25, 776-780. Yoshii, Y., Zhao, C., Zhao, K.D., Zobitz, M.E., An, K.N., Amadio, P.C., 2008. The effect of wrist position on the relative motion of tendon, nerve, and subsynovial connective tissue within the carpal tunnel in a human cadaver model. Journal of orthopaedic research : official publication of the Orthopaedic Research Society 26, 1153-1158.

Zech, S., Goesling, T., Hankemeier, S., Knobloch, K., Geerling, J., Schultz-Brunn, K., Krettek, C., Richter, M., 2006. Differences in the mechanical properties of calcaneal artificial specimens, fresh frozen specimens, and embalmed specimens in experimental testing. Foot \& ankle international. / American Orthopaedic Foot and Ankle Society [and] Swiss Foot and Ankle Society 27, 1126-1136.

Zuo, D., Zhou, Z., Wang, H., Liao, Y., Zheng, L., Hua, Y., Cai, Z., 2015. Endoscopic versus open carpal tunnel release for idiopathic carpal tunnel syndrome: a meta-analysis of randomized controlled trials. Journal of orthopaedic surgery and research 10, 12. 